\title{
The obstetric airway: things are seldom as they seem
}

\author{
M. Joanne Douglas, MD • Roanne L. Preston, MD
}

Published online: 1 April 2011

(C) Canadian Anesthesiologists' Society 2011

"Things are seldom what they seem,

Skim milk masquerades as cream..."

Gilbert and Sullivan, H.M.S. Pinafore

For decades, the obstetric airway struck fear in the heart of the anesthesiologist as the incidence of failed intubation in obstetrics was reported as 1:250 to 1:300, approximately ten times more frequent than in the general surgical population. ${ }^{1,2}$ In spite of our fancy airway tools, algorithms, and difficult airway workshops, a recently published experience of more than 1,000 obstetric general anesthetics (GA) suggested we had not made progress in this area: the reported incidence of failed intubation was 1:250, and difficult intubation occurred in $3.3 \%$ of cases. ${ }^{3}$

So, it is with some relief that one reads the article by McKeen et al. in which they report their experience with the maternal airway over a $20-y r$ period at a Canadian academic tertiary care hospital. ${ }^{4}$ In $2,633 \mathrm{GAs}$, the incidence of failed intubation was 1:1,300, similar to that of the general surgical population, and difficult intubation was encountered in $4.7 \%$, which is at the lower end of the reported range of $1.3 \%$ to $16.3 \%$ in obstetrics. ${ }^{3,5}$ Does this study refute the widely held belief that intubation is more difficult in the obstetric patient? In other words, do the results reflect the complete picture, or are they a case of "things are seldom what they seem"?

M. J. Douglas, MD · R. L. Preston, MD

Department of Anesthesiology, Pharmacology and Therapeutics, University of British Columbia, Vancouver, BC, Canada

M. J. Douglas, MD ( $\square)$

Department of Anesthesia, BC Women's Hospital, Rm 1Q72,

4500 Oak Street, Vancouver, BC V6H 3N1, Canada

e-mail: jdouglas@cw.bc.ca
The reasons for the apparent difficulty in managing the obstetric airway are both clear and unclear. Undoubtedly, there are anatomic and physiologic changes in pregnancy that make the airway of the term pregnant woman more likely to present a less-than-ideal view for intubation. Nevertheless, one would think we would have found solutions to manage the pregnant airway given the many alternatives to direct laryngoscopy, the development of obstetric-specific airway algorithms and associated training drills ${ }^{6}$ and efforts to ensure junior trainees are supervised when providing GA.

It is our view that there remains more to the story than solely the anatomical and physiological changes to the maternal airway - these factors could be overcome by the use of other airway devices. Other issues, e.g., the environment in which we provide GA in obstetrics, are more dominant and more difficult to tackle. General anesthesia usually is administered for an obstetric emergency, and under these circumstances, the available anesthesia assistance is less skilled, the atmosphere is tense, the time pressure is extreme (unlike anything else we face, other than perhaps a nasty trauma case), and our proclivity is to behave differently than we normally would for anesthesia in a high-risk patient. It is our view that the situational arena is probably the greatest key to our success or failure in managing the airway.

Compounding the problem is the fact that the GA rate in obstetrics is only $5 \%$ to $7 \%$ in most tertiary care academic centres. ${ }^{7}$ Consequently, a staff anesthesiologist may perform only five to ten GAs per year on average, resident trainees may perform even fewer, and nursing assistance, relied on by most units, may be turned to for only one GA per year. We have become so expert at providing neuraxial anesthesia that our experience in providing GA in obstetrics has diminished markedly. 
The declining use of GA in obstetrics has led to a concern that there will be a rise in the number of difficult/ failed intubations. ${ }^{5,8}$ Again, McKeen et al.'s study is reassuring since the incidence of difficult/failed intubation remained stable despite the decreased use of GA (from $5.2 \%$ to $1 \%$ ) over the 20 -year study period. ${ }^{4}$ Palanisamy et al. reported similar results in their study; i.e., a remarkable decline in GA rate from $4.5 \%$ to $0.6 \%$, (thanks to a concerted effort to avoid the obstetric airway) but no increase in the rate of difficult/failed intubation. ${ }^{9}$ In contrast to these two studies where, if possible, a GA was avoided, a review of 3,430 obstetric GAs from the UK over eight years showed no failed intubations and a difficult intubation rate of $1: 149 .^{10}$ The authors of the latter study suggest their success was attributable, in part, to having a high volume practice, readily "practicing" GA, when appropriate, and the presence of skilled anesthesia assistants. To summarize,we have two studies on the one hand that show a declining GA rate and, on the other, another study that shows a large volume of GAs, and all three studies show a difficult/failed intubation rate matching that of the general surgical population. Hence, other than the concern that the declining GA rate leads to trainees' lack of experience in the obstetric airway, these three studies give us hope that, in fact, our management of the obstetric airway has improved.

There are several possible reasons for this apparent improvement in obstetric airway management, the most likely being early identification of the difficult airway. ${ }^{4}$ Early airway assessment allows for establishment of a functioning epidural prior to the need for anesthesia for an operative delivery or for planning an alternate technique for intubation, such as awake intubation, should a GA be required. Unfortunately, the study by McKeen et al. does not provide that information. ${ }^{4}$ In fact, airway documentation was completed on only $16.8 \%$ of the charts of patients whose tracheas were intubated.

With the changing demographic characteristics of the obstetric patient, i.e., increasing maternal age and increasing body weight - both identified as risk factors in McKeen's study ${ }^{4}$ - the issues of appropriate airway assessment and subsequent anesthetic strategies become more critical. Hood et al. were at the forefront in drawing attention to an increased risk of difficult tracheal intubation in morbidly obese parturients. ${ }^{11}$ A large-scale Danish study showed a weak but significant correlation of body mass index to difficult tracheal intubation in non-obstetric obese subjects. ${ }^{12}$ Then again, Brodsky et al. found that obesity alone did not predict difficult intubation, whereas, large neck circumference and high Mallampati scores were predictors. ${ }^{13}$ When placed in the "ramped" position, the morbidly obese bariatric patient is easier to intubate; this technique should be used for all obese parturients. ${ }^{14}$
The study by McKeen et al. also points out the importance of vigilance in the postpartum period for women requiring GA. ${ }^{4}$ The only failed intubations occurred during anesthesia for postpartum tubal ligation, and only one of the two airways was predicted to be potentially difficult. Also, recent maternal mortality studies ${ }^{15,16}$ have pointed out the importance of the postpartum period as airway problems did not occur only at the time of induction (due to failed intubation), but also at the time of extubation or in the early postoperative period due to failure to reverse neuromuscular blockade adequately and to monitor patients appropriately.

How do we address the "other factors" that undoubtedly contribute to the difficult obstetric airway? The crisis mentality in the obstetric operating room when a GA is required results in less than ideal conditions for intubation. Consequences of time pressure include failure to place the patient in the optimum position for tracheal intubation and failure to assess the airway adequately. In addition, there is less than adequate assistance for cricoid pressure in many obstetric units, and the equipment may differ from that used in the general operating room. If ever there were a place for crisis resource management or use of simulation, it is in the setting of GA for the obstetric emergency. Simulation should help the anesthesiologist (and trainees) learn how to function efficiently during a crisis, and its use should be mandatory for all individuals practicing obstetric anesthesia. $^{7}$

The caveat regarding the results of McKeen et al. is their origin, i.e., a high-volume obstetrics unit where there is a wealth of experience and where the labour epidural rate has climbed steadily. ${ }^{4}$ In the last reported five-year epoch, the labour epidural rate was $80 \%$, hence, GA could be avoided more easily than in smaller centres without a 24-hr labour analgesia service. Also, their anesthesia department has established an excellent relationship with its obstetrical care providers, ensuring antepartum assessment of women with medical conditions, obesity, and previous anesthetic difficulties. Many anesthesia departments are challenged to provide such a good antepartum consultation service, not only from the resource perspective, but also from the perspective of receiving appropriate referrals from obstetrical colleagues.

So, can we claim, as the authors have claimed, that "intubation is not more difficult in the obstetric population"? ${ }^{4}$ Probably not. However, we can claim that the incidence of difficult/failed intubation in an obstetric population will be similar to that of other surgical populations under the following conditions: 24-hr in-house service by obstetric anesthesiologists, the opportunity for airway assessment either antepartum or early in labour, and appropriate planning, including avoidance of GA in a patient with a predicted difficult airway. The use of 
simulation of obstetric crises might address the situational anxiety that is likely a dominant factor in the persistent high rate of difficult/failed intubation in other centres. Hopefully, this preparation will eventually result in a decrease in airway problems associated with GA in the peripartum period. After all, GA will always be needed in obstetrics; therefore, we must continue to find ways to provide it in the safest fashion possible to ensure our trainees and experienced anesthesiologists are comfortable providing it when needed.

\section{Les voies aériennes en obstétrique: les apparences sont souvent trompeuses}

« Les apparences sont souvent trompeuses,

le lait écrémé se fait passer pour de la crème... » Gilbert et Sullivan, H.M.S. Pinafore

Pendant plusieurs décennies, les voies aériennes des patientes en obstétrique ont suscité la peur dans le cœur des anesthésiologistes. En effet, on a rapporté que l'incidence d'échec à l'intubation chez cette population se situait entre 1:250 et 1:300 cas, soit approximativement dix fois plus que chez la population chirurgicale générale. ${ }^{1,2}$ En dépit de nos outils compliqués de prise en charge des voies aériennes, de nos algorithmes et de nos ateliers sur les voies aériennes difficiles, une expérience récemment publiée portant sur plus de 1000 anesthésies générales (AG) en obstétrique suggérait que nous n'avons pas fait de progrès dans ce domaine : l'incidence rapportée d'échec était de 1:250, et l'intubation a été difficile dans 3,3\% des cas. ${ }^{3}$

C'est donc avec un certain soulagement que le lecteur prend connaissance de l'article de McKeen et coll., dans lequel les auteurs relatent leur expérience avec les voies aériennes en obstétrique au cours d'une période de 20 ans dans un hôpital universitaire canadien de soins tertiaires. ${ }^{4}$ Sur 2633 AG, l'incidence d'échec à l'intubation était de 1:1300, soit un taux semblable à celui rencontré dans la population chirurgicale en général, et l'intubation a été difficile dans $4,7 \%$ des cas, un chiffre qui se situe au bas de l'échelle de valeurs rapportées de $1,3 \%$ à $16,3 \%$ en obstétrique. ${ }^{3,5}$ Cette étude réfute-t-elle la conviction très répandue que l'intubation est plus difficile chez une patiente en obstétrique? En d'autres mots, ces résultats sont-ils le reflet de la réalité, ou sont-ils un cas où « il ne faut pas se fier aux apparences »?

Les raisons derrière la difficulté apparente de prise en charge des voies aériennes de la population obstétricale sont à la fois évidentes et peu claires. Il ne fait aucun doute que certains changements anatomiques et physiologiques survenant au cours de la grossesse rendent les voies aériennes de la femme enceinte à terme plus susceptibles de s'éloigner d'une vue idéale pour l'intubation. Toutefois, il serait logique qu'on ait trouvé des solutions pour prendre en charge les voies aériennes des femmes enceintes, étant donné les nombreuses alternatives existantes à la laryngoscopie directe, en plus de la mise au point d'algorithmes pour les voies aériennes spécifiques à l'obstétrique, les sessions de formation associées ${ }^{6}$ et les efforts pour garantir que les stagiaires en début de formation sont supervisés lorsqu'ils font une AG.

Selon nous, les changements anatomiques et physiologiques survenant au niveau des voies aériennes pendant la grossesse ne sont qu'une partie des facteurs qui rendent l'intubation problématique chez cette population, car ces facteurs seuls pourraient être facilement contrôlés en utilisant d'autres dispositifs de prise en charge des voies aériennes. D'autres éléments, par exemple l'environnement dans lequel l'AG est réalisée en obstétrique, sont plus prépondérants et plus difficiles à maîtriser. L'anesthésie générale est administrée, en règle générale, pour une urgence obstétricale; dans de telles circonstances, les personnes nous assistant en salle d'opération sont souvent moins compétentes, l'ambiance est tendue, la contrainte de temps est extrême (à l'inverse de tous les autres cas auxquels nous sommes confrontés, à l'exception peut-être d'un cas particulièrement compliqué de traumatisme) - tous ces facteurs nous incitent à nous comporter différemment de ce que nous ferions habituellement pour réaliser une anesthésie chez un patient à risque élevé. Selon nous, le contexte et la situation sont probablement les principales clés de notre succès - ou de notre échec - à prendre en charge ces voies aériennes.

À ce problème s'ajoute le fait que le taux d'AG en obstétrique n'est que de $5 \%$ à $7 \%$ dans la plupart des hôpitaux universitaires de soins tertiaires. ${ }^{7}$ En d'autres termes, un anesthésiologiste travaillant dans un tel centre pourrait ne réaliser que cinq à dix AG par année en moyenne chez cette population, les résidents encore moins, et dans le personnel infirmier, dont l'assistance est cruciale dans la plupart des unités, une personne pourrait n'assister qu'à une AG par année. Nous sommes devenus tellement experts en anesthésie neuraxiale que notre expérience en matière d'AG dans un contexte obstétrical a diminué de façon notable.

Le recours de plus en plus rare à l'AG en obstétrique a suscité des inquiétudes quant à l'augmentation du nombre d'intubations difficiles ou impossibles. ${ }^{5,8}$ Mais l'étude de McKeen et coll. est, une fois de plus, rassurante : selon leurs données, l'incidence d'intubation difficile ou impossible est demeurée stable malgré la réduction du nombre d'AG (de 5,2\% à $1 \%$ ) au cours des vingt années à 
l'étude. ${ }^{4}$ Palanisamy et coll. ont rapporté des résultats similaires dans leur étude, soit un déclin notable du nombre d'AG, passant de $4,5 \%$ à $0,6 \%$ (en raison d'un effort concerté d'éviter la prise en charge des voies aériennes chez la population obstétricale), mais pas d'augmentation $\mathrm{du}$ taux d'intubation difficile ou impossible. ${ }^{9}$ À la différence de ces deux études où, dans la mesure du possible, on a évité de réaliser une $\mathrm{AG}$, dans un compte rendu de 3430 AG obstétricales au Royaume-Uni sur une durée de huit ans, il n'y a pas eu d'échec d'intubation et le taux d'intubation difficile était de $1: 149 .{ }^{10}$ Les auteurs de cette étude suggèrent que leur succès était attribuable au moins en partie au volume élevé de leur pratique, à leur propension à « réaliser » une AG, lorsque nécessaire, et à la présence d'assistants en anesthésie compétents. Pour résumer : nous disposons, d'une part, de deux études qui montrent un taux d'AG sur le déclin et, d'autre part, d'une autre étude montrant un volume important d'AG, et ces trois études démontrent toutes un taux d'intubation difficile ou d'échec correspondant à celui rencontré dans la population chirurgicale en général. Dès lors, hormis l'inquiétude qu'une réduction dans le nombre d'AG réalisées entraîne des lacunes dans l'expérience des personnes en formation en matière de voies aériennes obstétricales, ces trois études nous font espérer que notre prise en charge des voies aériennes pour la population obstétricale s'est en fait améliorée.

Il existe plusieurs raisons possibles pour expliquer cette amélioration apparente dans la prise en charge des voies aériennes en obstétrique, la plus plausible étant l'identification précoce des voies aériennes difficiles. ${ }^{4}$ Une évaluation précoce des voies aériennes permet d'installer une péridurale fonctionnelle avant l'AG pour un accouchement en salle d'opération ou de prévoir une autre technique d'intubation, telle que l'intubation éveillée, si une AG s'avérait nécessaire. Malheureusement, l'étude de McKeen et coll. ne nous fournit pas de tels renseignements. ${ }^{4}$ En fait, la documentation sur les voies aériennes n'était complète que dans $16,8 \%$ des dossiers des patientes ayant subi une intubation trachéale.

Au vu de l'évolution des caractéristiques démographiques de la clientèle obstétricale, soit une augmentation de l'âge maternel et du poids, deux caractéristiques identifiées comme étant des facteurs de risque dans l'étude de McKeen, ${ }^{4}$ les questions concernant l'évaluation adéquate des voies aériennes et des stratégies pour une anesthésie subséquente deviennent encore plus cruciales. Hood et coll. ont été parmi les premiers à attirer l'attention sur un risque accru d'intubation trachéale difficile chez les parturientes obèses morbides. ${ }^{11}$ Une étude danoise à grande échelle a révélé une corrélation, faible mais significative, entre l'indice de masse corporelle et l'intubation trachéale difficile chez des patients obèses non obstétricaux. ${ }^{12}$ Par contre, Brodsky et coll. ont découvert que l'obésité seule ne suffisait pas à prédire une intubation difficile, alors qu'une circonférence importante du cou et des scores de Mallampati élevés constituaient des prédicteurs indépendants. ${ }^{13}$ Les patients de chirurgie bariatrique obèses morbides souffrant d'apnée obstructive du sommeil devraient être placés en position 'inclinée' afin de faciliter l'intubation trachéale; en fait, cette technique devrait être utilisée pour tous les patients obèses. ${ }^{14}$

L'étude de McKeen et coll. souligne également l'importance de la vigilance en période post-partum chez les femmes ayant besoin d'une $\mathrm{AG}^{4}{ }^{4}$ Les seuls échecs d'intubation mentionnés dans l'étude sont survenus pendant une anesthésie pour des ligatures tubaires post-partum, et les voies aériennes ont été anticipées comme potentiellement difficiles d'intubation dans un de ces deux cas. En outre, de récentes études de mortalité maternelle ${ }^{15,16}$ ont mis en exergue l'importance de la période post-partum : en effet, les problèmes au niveau des voies aériennes ne surviennent pas seulement au moment de l'induction (en raison de l'échec de l'intubation). Des problèmes au niveau des voies aériennes sont également apparus au moment de l'extubation ou au début de la période postopératoire en raison d'une mauvaise neutralisation du bloc neuromusculaire ou d'un monitorage inadapté des patientes.

Comment peut-on aborder les « autres facteurs » qui contribuent certainement à créer des voies aériennes obstétricales difficiles? La mentalité de crise dans la salle d'opération obstétricale lorsqu'une AG est nécessaire crée des conditions d'intubation qui sont loin d'être idéales. La contrainte de temps peut avoir pour conséquences le mauvais positionnement de la patiente pour réaliser une intubation trachéale et une mauvaise évaluation des voies aériennes. De plus, l'assistance pour la pression cricoïdienne est loin d'être adaptée dans plusieurs unités obstétricales, et le matériel pourrait être différent de ce qu'on utilise dans une salle d'opération habituelle. S'il existe un endroit où la gestion des ressources en période de crise ou le recours à la simulation pourraient être utiles, c'est bien dans le contexte d'une AG pour une urgence obstétricale. La simulation devrait aider l'anesthésiologiste (et les résidents) à apprendre comment être efficace pendant une crise; son utilisation devrait être obligatoire pour toutes les personnes faisant de l'anesthésie obstétricale. $^{7}$

La seule réserve concernant les résultats de l'étude de McKeen et coll. est liée à leur origine, c'est-à-dire une unité obstétricale à haut volume où l'expérience est abondante et le taux de péridurales pour le travail obstétrical est en croissance constante. ${ }^{4} \mathrm{Au}$ cours des cinq dernières années de la période rapportée, le taux de péridurales pour le travail obstétrical était de $80 \%$, ce qui a permis d'éviter plus facilement l'AG que dans des centres de moindre envergure qui ne disposent pas d'un service d'analgésie pour le travail obstétrical 24 heures par jour. En outre, le département 
d'anesthésie de ces auteurs a établi d'excellentes relations avec les fournisseurs de soins obstétricaux, ce qui garantit une évaluation ante partum des femmes souffrant de conditions médicales, d'obésité ou ayant rencontré des difficultés avec l'anesthésie par le passé. Pour nombre de départements d'anesthésie, fournir un service de consultation ante partum d'une telle qualité représente un défi, non seulement en termes de ressources, mais également au niveau des consultations de la part de leurs collègues en obstétrique.

Peut-on dès lors affirmer, comme les auteurs de cette étude, que "l'intubation n'est pas plus difficile chez la population obstétricale » $?^{4}$ Probablement pas. Toutefois, nous pouvons affirmer que l'incidence d'intubation difficile ou impossible, chez une population obstétricale, est semblable à celle d'autres populations chirurgicales si les conditions suivantes sont remplies : un service à l'interne, 24 heures par jour, offert par des anesthésiologistes obstétricaux; la possibilité d'évaluer les voies aériennes soit ante partum, soit au début du travail obstétrical; et une bonne planification, notamment la non-réalisation d'une AG chez une patiente avec des voies aériennes anticipées comme difficiles. Le recours à la simulation dans les crises en obstétrique pourrait dissiper l'anxiété liée à la situation, probablement un facteur dominant expliquant le taux élevé constant d'intubations difficiles ou impossibles dans d'autres centres. Avec un peu de chance, la préparation des anesthésiologistes résultant du recours à la simulation aura comme conséquence ultime une réduction des problèmes de voies aériennes associés à l'AG en période périnatale. Après tout, l'AG sera toujours nécessaire en obstétrique; par conséquent, nous devons continuer de trouver des façons de la procurer de la manière la plus sécuritaire qui soit afin de garantir que les anesthésiologistes en formation et les anesthésiologistes d'expérience la fournissent en toute aise lorsqu'elle est nécessaire.

Competing interests None declared.

\section{References}

1. Lyons $G$. Failed intubation. Six years' experience in a teaching maternity unit. Anaesthesia 1985; 40: 759-62.
2. Hawthorne L, Wilson R, Lyons G, Dresner M. Failed intubation revisited: 17-yr experience in a teaching maternity unit. $\mathrm{Br} \mathrm{J}$ Anaesth 1996; 76: 680-4.

3. McDonnell NJ, Paech MJ, Clavisi OM, Scott KL, ANZCA Trials Group. Difficult and failed intubation in obstetric anaesthesia: an observational study of airway management and complications associated with general anaesthesia for caesearean section. Int $\mathbf{J}$ Obstet Anesth 2008; 17: 292-7.

4. McKeen DM, George RB, O'Connell CM, et al. Difficult and failed intubation: Incident rates and maternal, obstetrical and anesthetic predictors. Can J Anesth 2011; 58. DOI:10.1007/ s12630-011-9491-9.

5. Tsen LC, Pitner R, Camann WR. General anesthesia for cesarean section at a tertiary care hospital 1990-1995: indications and implications. Int J Obstet Anesth 1998; 7: 147-52.

6. Mhyre JM, Healy D. Focused review: the unanticipated difficult intubation in obstetrics. Anesth Analg 2011; 112: 648-52.

7. Lipman S, Carvalho B, Brock-Utne J. The demise of general anesthesia in obstetrics revisited: prescription for a cure. Int $\mathbf{J}$ Obstet Anesth 2005; 14: 2-4.

8. Russell $R$. Failed intubation in obstetrics: a self- fulfilling prophesy? Int J Obstet Anesth 2007; 16: 1-3.

9. Palanisamy A, Mitani AA, Tsen LC. General anesthesia for cesarean delivery at a tertiary care hospital from 2000 to 2005: a retrospective analysis and 10-year update. Int J Obstet Anesth 2011; 20: 10-6.

10. Djabatey EA, Barclay PM. Difficult and failed intubation in 3430 obstetric general anaesthetics. Anaesthesia 2009; 64: 1168-71.

11. Hood DD, Dewan DM. Anesthetic and obstetric outcome in morbidly obese parturients. Anesthesiology 1993; 79: 1210-8.

12. Lundstrom LH, Moller AM, Rosenstock C, et al. High body mass index is a weak predictor for difficult and failed tracheal intubation: a cohort study of 91, 332 consecutive patients scheduled for direct laryngoscopy registered in the Danish Anesthesia Database. Anesthesiology 2009; 110: 266-74.

13. Brodsky JB, Lemmens HJ, Brock-Utne JG, Vierra M, Saidman $L J$. Morbidy obesity and tracheal intubation. Anesth Analg 2002; 94: 732-6.

14. Neligan PJ, Porter S, Max B, Malhotra G, Greenblatt EP, Ochroch EA. Obstructive sleep apnea is not a risk factor for difficult intubation in morbidly obese patients. Anesth Analg 2009; 109: 1182-6.

15. Hawkins JL, Chang J, Palmer SK, Gibbs CP, Callaghan WM. Anesthesia-related maternal mortality in the United States: 1979-2002. Obstet Gynecol 2011; 117: 69-74.

16. Saving Mother's Lives; Reviewing maternal deaths to make motherhood safer - 2003-2005. London: Confidential Enquiry into Maternal and Child Health, 2007. Available from URL: http://www.cemach.org.uk/getattachment/26dae364-1fc9-4a29a6cb-afb3f251f8f7/Saving-Mothers\%20-Lives-2003-2005-(Fullreport).aspx (accessed March 2011). 